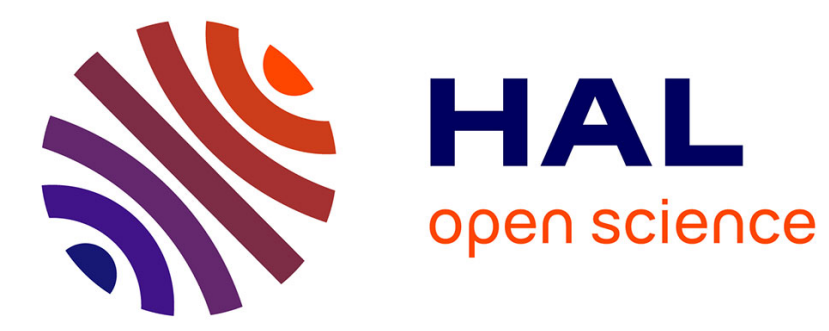

\title{
On Notions of Output Finite-Time Stability
}

Konstantin Zimenko, Denis Efimov, Andrey Polyakov, Artem Kremlev

\section{To cite this version:}

Konstantin Zimenko, Denis Efimov, Andrey Polyakov, Artem Kremlev. On Notions of Output FiniteTime Stability. Proc. European Control Conference, Jun 2019, Naples, Italy. hal-02084981

\section{HAL Id: hal-02084981 \\ https://hal.inria.fr/hal-02084981}

Submitted on 30 Mar 2019

HAL is a multi-disciplinary open access archive for the deposit and dissemination of scientific research documents, whether they are published or not. The documents may come from teaching and research institutions in France or abroad, or from public or private research centers.
L'archive ouverte pluridisciplinaire HAL, est destinée au dépôt et à la diffusion de documents scientifiques de niveau recherche, publiés ou non, émanant des établissements d'enseignement et de recherche français ou étrangers, des laboratoires publics ou privés. 


\title{
On Notions of Output Finite-Time Stability
}

\author{
Konstantin Zimenko ${ }^{\dagger}$, Denis Efimov ${ }^{\ddagger, \dagger}$, Andrey Polyakov ${ }^{\ddagger, \dagger}$, and Artem Kremlev ${ }^{\dagger}$
}

\begin{abstract}
Lyapunov characterizations of output finite-time stability are presented for the system $\dot{x}=f(x), y=h(x)$ which is locally Lipschitz continuous out of the set $\mathcal{Y}=\{x \in$ $\left.\mathbb{R}^{n}: h(x)=0\right\}$ and continuous on $\mathbb{R}^{n}$. The definitions are given in the form of $\mathcal{K}$ and $\mathcal{K} \mathcal{L}$ functions. Necessary and sufficient conditions for output finite-time stability are given using Lyapunov functions. The theoretical results are supported by numerical examples.
\end{abstract}

\section{INTRODUCTION}

The output stability analysis is continuing to be the subject of numerous studies in recent years (see, for example, [1][6], etc.). This attention is motivated by a number of technical systems, where one is only concerned with stability and stabilizability of the output variables instead of the full set of state variables. In addition, partial stability (see, for example, [7]-[10], [17], etc.) is a particular case of output stability, where the output is a subset of the state. The output stability analysis is required in a number of applications including both regulation (for instance, the use of adaptive control techniques, spacecraft stabilization [9], drift of the gyroscope axis [9], etc.) and observer design problems (for instance, observer designs where the output variables represent the observation error).

For locally Lipschitz continuous systems

$$
\dot{x}=f(x, u), \quad y=h(x), \quad x(0)=x_{0}
$$

the papers [11]-[16] develop a theoretical framework of Input-to-Output Stability (IOS), that have risen in Input-toState Stability. The notion of Input-to-Output Stability is devoted to robust output stability with respect to disturbances (inputs) $u$. In addition, the papers [13], [14] provide the results on uniform output stability (UOS) with respect to inputs from an admissible set (or output uniform global asymptotic stability (oUGAS) in some references), i.e. the system output $y$ tends to 0 as $t \rightarrow \infty$. Note, that this framework is given in terms of necessary and sufficient Lyapunov characterizations.

The present paper is devoted to Output Finite-Time Stability (OFTS) for the systems in the case of absence of inputs (disturbances). This notion implies completion of output

\footnotetext{
*This work is supported by the Russian Science Foundation under grant 17-19-01422.

${ }^{\dagger}$ Konstantin Zimenko, Andrey Polyakov, Denis Efimov and Artem Kremlev are with Faculty of Control Systems and Robotics, ITMO University, 49 Kronverkskiy av., 197101 Saint Petersburg, Russia.

${ }^{\ddagger}$ Andrey Polyakov and Denis Efimov are with Inria, Univ. Lille, CNRS, UMR 9189 - CRIStAL, F-59000 Lille, France.

e-mail: kostyazimenko@gmail.com, denis.efimoveinria.fr, andrey.polyakoveinria.fr, kremlev_artememail.ru
}

transients in a finite time, i.e. the output $y\left(t, x_{0}\right)=0$ for all $t \geq T_{0}$ and some $0 \leq T_{0}<+\infty$. Such system behaviour is needed in many control applications. Considering the observation problems, a convergence of observed states to the real ones in finite time is always preferable.

Note, that despite the names similarity of OFTS (or IOS) and Input-Output Finite-Time Stability (IO-FTS) (as, for example, presented in [19]), these notions are independent concepts, since the latter one implies that the output do not exceed an assigned threshold during a specified time interval.

The results presented in this paper are related to the class of systems that are locally Lipschitz out of the set $\mathcal{Y}=$ $\left\{x \in \mathbb{R}^{n}: h(x)=0\right\}$ and continuous everywhere. Since this class is wider than considered in [13], [14], firstly, we extended the results on necessary and sufficient Lyapunov characterizations of OS for the system under consideration. Afterwards, the paper provides the necessary and a number of sufficient conditions to ensure OFTS. It is noteworthy that in some cases an OFTS-Lyapunov function can be chosen in less restrictive form in comparison with Lyapunov functions presented in [13], [14].

The paper is organized in the following way. Notation used in the paper is given in Section II. Section III presents the system under consideration and recalls basics on OS and OFTS. Section IV presents the main result on necessary and sufficient conditions for OFTS with examples. Finally, concluding remarks are given in Section V.

\section{NOTATION}

Through the paper the following notation will be used:

- $\mathbb{R}^{n}$ denotes the $n$ dimensional Euclidean space with vector norm $|\cdot|$;

- $\mathbb{R}_{>0}=\{x \in \mathbb{R}: x>0\}, \mathbb{R}_{\geq 0}=\{x \in \mathbb{R}: x \geq 0\}$ where $\mathbb{R}$ is the field of real numbers;

- A continuous function $\sigma: \mathbb{R}_{\geq 0} \rightarrow \mathbb{R}_{\geq 0}$ belongs to class $\mathcal{K}$ if it is strictly increasing and $\sigma(0)=0$. It belongs to class $\mathcal{K}_{\infty}$ if it is also radially unbounded;

- A continuous function $\beta: \mathbb{R}_{\geq 0} \times \mathbb{R}_{\geq 0} \rightarrow \mathbb{R}_{\geq 0}$ belongs to class $\mathcal{K} \mathcal{L}$ if $\beta(\cdot, r) \in \mathcal{K}$ and $\beta(r, \cdot)$ is decreasing to zero for any fixed $r \in \mathbb{R}_{\geq 0}$;

- For any real number $\alpha \in \mathbb{R}_{\geq 0}$ and for all real $x$ we set

$$
\lfloor x\rceil^{\alpha}=\operatorname{sign}(x)|x|^{\alpha} .
$$

\section{PRELIMINARIES}

\section{A. System under Consideration}

Consider the system of the type

$$
\dot{x}=f(x), \quad y=h(x)
$$


with states $x \in \mathbb{R}^{n}$ and outputs $y \in \mathbb{R}^{p}$.

Assumption 1 The vector field $f: \mathbb{R}^{n} \rightarrow \mathbb{R}^{n}$ ensures forward existence and uniqueness of the system solutions at least locally in time, $f(0)=0$.

Assumption 2 The function $h: \mathbb{R}^{n} \rightarrow \mathbb{R}^{p}$ is a continuously differentiable, $h(0)=0$ and there exists $\gamma \in \mathcal{K}$ such that $|h(x)| \leq \gamma(|x|)$ for all $x \in \mathbb{R}^{n}$.

Assumption 3 Let the vector field $f \in \mathcal{C}\left(\mathbb{R}^{n}\right)$ is locally Lipschitz continuous on $\mathbb{R}^{n} \backslash \mathcal{Y}$, where $\mathcal{Y}=\left\{x \in \mathbb{R}^{n}\right.$ : $h(x)=0\}$.

For the initial conditions $x_{0} \in \mathbb{R}^{n}$, let $\Phi\left(t, x_{0}\right)$ be a unique solution of the system (1) defined over a finite interval $\left[0, T_{s}\right)$ with some $T_{s}>0, y\left(t, x_{0}\right)=h\left(\Phi\left(t, x_{0}\right)\right)$.

\section{B. Output Stability}

The preliminaries in this subsection are based on theoretical framework of IOS and UOS presented in [11]-[16].

Deffinition 1 [13], [12] A system is forward complete if each $x_{0} \in \mathbb{R}^{n}$ produces a solution $\Phi\left(t, x_{0}\right)$ which is defined on $[0, \infty)$.

Deffinition 2 [11] The system (1) has the unboundedness observability $(U O)$ property if

$$
\limsup _{t \rightarrow T_{s}}\left|y\left(t, x_{0}\right)\right|=+\infty
$$

necessarily follows for each $x_{0} \in \mathbb{R}^{n}$ such that $T_{s}<+\infty$.

In other words, any unboundedness of the state vector can be observed using the output $y$. Hence, if the output is known to be bounded (which is the case under the output stability properties described below) then the UO property is equivalent to forward completeness [13]. Note, that any system has the property of UO in output $h(x)=x$.

Definition 3 [14], [15] A system (1) is output stable if

- it is forward complete, and

- there exists a $\mathcal{K} \mathcal{L}$-function $\beta$ such that

$$
\left|y\left(t, x_{0}\right)\right| \leq \beta\left(\left|x_{0}\right|, t\right) \quad \forall t \geq 0
$$

holds for all $x_{0} \in \mathbb{R}^{n}$.

If, in addition, there exists $\sigma \in \mathcal{K}$ such that

$$
\left|y\left(t, x_{0}\right)\right| \leq \sigma\left(\left|h\left(x_{0}\right)\right|\right) \quad \forall t \geq 0
$$

holds for all trajectories of the system, then the system is output-Lagrange output stable (OLOS). Finally, if one strengthens (2) to

$$
\left|y\left(t, x_{0}\right)\right| \leq \beta\left(\left|h\left(x_{0}\right)\right|, t\right), \quad \forall t \geq 0
$$

holding for all trajectories of the system, then the system is state-independent output stable (SIOS).

Lemma 1 [16] For system (1) having the UO property, the following relations are valid:

$$
S I O S \Rightarrow O L O S \Rightarrow I O S .
$$

In the general case, all inverse relations are not satisfied.

Let us present definitions for corresponded Lyapunov functions.
Definition 4 [12], [13] For the system (1), a smooth function $V$ and a function $\lambda: \mathbb{R}^{n} \rightarrow \mathbb{R}_{>0}$ are called respectively an OS-Lyapunov function and an auxiliary modulus if there exist $\alpha_{1}, \alpha_{2} \in \mathcal{K}_{\infty}$ so that

$$
\alpha_{1}(|h(x)|) \leq V(x) \leq \alpha_{2}(|x|) \quad \forall x \in \mathbb{R}^{n}
$$

holds and there exists $\alpha_{3} \in \mathcal{K} \mathcal{L}$ such that

$$
\dot{V}(x) \leq-\alpha_{3}(V(x), \lambda(x))
$$

for all $x \in \mathcal{X}$, where $\mathcal{X}=\left\{x \in \mathbb{R}^{n}: V(x)>0\right\}$, and either

(a) $\lambda$ satisfies the following conditions:

- $0 \leq \lambda(x) \leq|x|$ for all $x \in \mathbb{R}^{n}$;

- $\lambda$ is locally Lipschitz on the set $\mathcal{X}$ and satisfies

$$
D \lambda(x) f(x) \leq 0
$$

for almost all $x \in \mathcal{X}$,

or

(b) there exists some $\theta \in \mathcal{K}$ such that

$$
\lambda\left(\Phi\left(t, x_{0}\right)\right) \leq \theta\left(\left|x_{0}\right|\right)
$$

for all $t \geq 0$ and $x \in \mathcal{X}$.

The function $V$ is called an OLOS-Lyapunov function if it is an OS-Lyapunov function, and in addition, inequality (5) can be strengthened to

$$
\alpha_{1}(|h(x)|) \leq V(x) \leq \alpha_{2}(|h(x)|), \quad \forall x \in \mathbb{R}^{n} .
$$

The function $V$ is called the SIOS-Lyapunov function if the inequality (9) is satisfied and there exists $\alpha_{3} \in \mathcal{K}$ such that for all $x \in \mathcal{X}$ :

$$
\dot{V}(x) \leq-\alpha_{3}(V(x)) .
$$

An auxiliary modulus $\lambda$ satisfying property (a) is called a strong auxiliary modulus, and one satisfying property (b) is a weak auxiliary modulus [13].

Note that in the case of OLOS- or SIOS-Lyapunov function we have $\mathcal{X}=\mathbb{R}^{n} \backslash \mathcal{Y}$.

Remark 1 [13] Observe that if a system is Lyapunov stable (i.e. $\left|\Phi\left(t, x_{0}\right)\right| \leq \alpha\left(\left|x_{0}\right|\right)$ for all $t \geq 0$ and some $\alpha \in \mathcal{K})$, then the function $\lambda(x)=|x|$ is a weak auxiliary modulus.

Note that given above definitions and an analogue of Theorem 2 given below are presented in [11]-[16] for the dynamical system $\dot{x}=f(x, u), y=h(x)$ with locally Lipschitz continuous $f, h$ in the sense of uniform stability with respect to inputs $u$. Despite this, all given definitions remain valid for the class of systems under consideration, and the proof of the Lemma 1 is a direct consequence of Definition 3. 


\section{Output Finite-Time Stability}

Now let us present the definitions on output finite-time stability.

Definition 5 The system (1) is said to be OFTS if it is OS and for any $x_{0} \in \mathbb{R}^{n}$ there exists $0 \leq T_{0}<+\infty$ such that $y\left(t, x_{0}\right)=0$ for all $t>T_{0}$. The function $T\left(x_{0}\right)=\inf \left\{T_{0} \geq\right.$ $\left.0: y\left(t, x_{0}\right)=0 \forall t \geq T_{0}\right\}$ is called the settling-time function.

The paper [17] deals with partial finite-time stability that is a particular case of OFTS, where $h$ is a projection on a subspace of the state space $\mathbb{R}^{n}$.

Theorem 1 [17] Consider the system

$$
\begin{array}{ll}
\dot{x}_{1}=f_{1}\left(x_{1}, x_{2}\right), & x_{1}(0)=x_{10}, \\
\dot{x}_{2}=f_{2}\left(x_{1}, x_{2}\right), & x_{2}(0)=x_{20},
\end{array}
$$

where $x_{1} \in \mathcal{N} \subseteq \mathbb{R}^{n_{1}}, x_{2} \in \mathbb{R}^{n_{2}}$ are the states, $f_{1}$ : $\mathcal{N} \times \mathbb{R}^{n_{2}} \rightarrow \mathbb{R}^{n_{1}}$ and $f_{2}: \mathcal{N} \times \mathbb{R}^{n_{2}} \rightarrow \mathbb{R}^{n_{2}}$ are such that, for every $\left(x_{1}, x_{2}\right) \in \mathcal{N} \times \mathbb{R}^{n_{2}}, f_{1}\left(0, x_{2}\right)=0$ and $f_{1}(\cdot, \cdot)$, $f_{2}(\cdot, \cdot)$ are jointly continuous in $x_{1}$ and $x_{2}$. If there exist a continuously differentiable function $V: \mathcal{N} \times \mathbb{R}^{n_{2}} \rightarrow \mathbb{R}$, a class $\mathcal{K}$ function $\alpha(\cdot)$, a continuous function $k:[0,+\infty) \rightarrow$ $\mathbb{R}_{>0}$, a real number $\mu \in(0,1)$ and an open neighbourhood $\mathcal{M} \subseteq \mathcal{N}$ of $x_{1}=0$ such that for $\left(x_{1}, x_{2}\right) \in \mathcal{M} \times \mathbb{R}^{n_{2}}$

$$
\begin{gathered}
V\left(0, x_{2}\right)=0, \\
\alpha\left(\left|x_{1}\right|\right) \leq V\left(x_{1}, x_{2}\right), \\
\dot{V}\left(x_{1}, x_{2}\right) \leq-k\left(\left|x_{2}\right|\right)\left(V\left(x_{1}, x_{2}\right)\right)^{\mu}
\end{gathered}
$$

then (11) is finite-time stable with respect to $x_{1}$ (OFTS with $\left.y=x_{1}\right)$. Moreover, there exist a neighbourhood $\mathcal{N}_{0}$ of $x_{1}=0$ and a settling-time function $T: \mathcal{N}_{0} \times \mathbb{R}^{n_{2}} \rightarrow[0, \infty)$ such that

$$
T\left(x_{10}, x_{20}\right) \leq q^{-1}\left(\frac{\left(V\left(x_{10}, x_{20}\right)\right)^{1-\mu}}{1-\mu}\right),\left(x_{10}, x_{20}\right) \in \mathcal{N}_{0} \times \mathbb{R}^{n_{2}},
$$

where $q:[0, \infty) \rightarrow \mathbb{R}$ is continuously differentiable and satisfies

$$
\dot{q}(t)=k\left(\left|x_{2}(t)\right|\right), \quad q(0)=0, \quad t \geq 0,
$$

and $T(\cdot, \cdot)$ is jointly continuous on $\mathcal{N}_{0} \times \mathbb{R}^{n_{2}}$.

Comparing with Lyapunov characterizations of output stability given in Definition 4, the condition (14) is rather restrictive.

\section{MAIN RESULT}

In this section we will assume that assumptions 1-3 are satified, then the system under consideration (1) is of wider class then in [13] and [14] (the Lipschitz continuity may be violated on $\mathcal{Y}$ ). The following theorem extends results of these papers and gives the necessary and sufficient Lyapunov characterizations of output stability for the system (1).

Theorem 2 Suppose the system (1) is UO.

(1) The following claims are equivalent for the system:

(a) it is OLOS;

(b) it admits an OLOS-Lyapunov function with a weak auxiliary modulus;

(c) it admits an OLOS-Lyapunov function with a strong auxiliary modulus.
(2) The following claims are equivalent for the system:

(a) it is SIOS;

(b) it admits an SIOS-Lyapunov function.

Based on given characterizations we may obtain the following results on OFTS.

Theorem 3 Consider the UO system (1). The following properties are equivalent:

1) the system is OFTS satisfying the condition (3);

2) there exists a smooth OLOS-Lyapunov function $V$ : $\mathbb{R}^{n} \rightarrow \mathbb{R}_{\geq 0}$ for the system (1) satisfying for all $x \in \mathbb{R}^{n}$

$$
\int_{0}^{V(x)} \frac{d s}{-\dot{V}\left(\Phi\left(\theta_{x}(s), x\right)\right)}<+\infty,
$$

where $\theta_{x}$ is the inverse of $t \mapsto V(\Phi(t, x))$.

According to [14], for any $\alpha_{3} \in \mathcal{K} \mathcal{L}$, there exist $\kappa_{1}, \kappa_{2} \in$ $\mathcal{K}$ such that

$$
\alpha_{3}(s, t) \geq \frac{\kappa_{1}(s)}{1+\kappa_{2}(t)}
$$

for all $s, t \in \mathbb{R}_{\geq 0}$. Hence, for OLOS-Lyapunov function we have

$$
\dot{V}(x) \leq-\frac{\kappa_{1}(V(x))}{1+\kappa_{2}(\lambda(x))}
$$

and the following corollary to Theorem 3 on sufficient condition of OFTS can be given.

Corollary 1 Consider the OLOS system (1). Let $V$ is an OLOS-Lyapunov function satisfying the inequality

$$
\int_{0}^{V\left(x_{0}\right)} \frac{d s}{\kappa_{1}(s)}<+\infty,
$$

with $\kappa_{1} \in \mathcal{K}$ as in (16). Then the system (1) is OFTS.

Finding an OLOS-Lyapunov function with a weak or strong auxiliary modulus is a difficult task in some cases. Let us give the definition for a local auxiliary modulus.

Definition 6 An auxiliary modulus $\lambda$ in (6) is called local auxiliary modulus if there exists some (jointly) continuous function $\varrho: \mathbb{R}_{\geq 0} \times \mathbb{R}_{\geq 0} \rightarrow \mathbb{R}_{\geq 0}$ such that $\varrho(\cdot, t)$ is nondecreasing, $\varrho(s, \cdot) \in \overline{\mathcal{K}}$ and for any $T \in \mathbb{R}_{\geq 0}$

$$
\lambda\left(\Phi\left(t, x_{0}\right)\right) \leq \varrho\left(\left|x_{0}\right|, T\right)
$$

for all $t \in[0, T]$ and $x \in \mathcal{X}$.

Using Definition 6 the following result can be given.

Lemma 2 Consider the UO system (1). Let there exists a smooth Lyapunov function $V: \mathbb{R}^{n} \rightarrow \mathbb{R}_{\geq 0}$ for the system (1) satisfying the conditions (6) and (9) with a local auxiliary modulus $\lambda$. If for any $\varepsilon \in \mathbb{R}_{\geq 0}$ there exists $T_{\varepsilon} \in \mathbb{R}_{\geq 0}$ such that

$$
\int_{0}^{\alpha_{2} \circ \gamma(\varepsilon)} \frac{d s}{\alpha_{3}\left(s, \varrho\left(\varepsilon, T_{\varepsilon}\right)\right)} \leq T_{\varepsilon}<+\infty,
$$

then the system (1) is OFTS. Moreover,

$$
T\left(x_{0}\right) \leq T_{\varepsilon}
$$

for $\left\{x_{0} \in \mathbb{R}^{n}:\left|x_{0}\right| \leq \varepsilon\right\}$.

Remark 2 Note that local auxiliary modulus is less restrictive than weak auxiliary modulus (8), that makes the search for the OFTS-Lyapunov function (9), (6), (18) even simpler in comparison with the OLOS-Lyapunov function 
presented in Definition 4, i.e. the strong or weak auxiliary modulus has to be bounded on the system trajectories, while a local auxiliary modulus can be just a sufficiently slowly growing function of time.

Using the inequality (16) the following corollary on Lemma 2 can be given.

Corollary 2 Consider the system (1) having UO. Let there exists a smooth Lyapunov function $V: \mathbb{R}^{n} \rightarrow \mathbb{R}_{\geq 0}$ as in Lemma 2. The system (1) is OFTS if for any $\varepsilon \in \mathbb{R}_{\geq 0}$ there exists $T_{\varepsilon} \in \mathbb{R}_{\geq 0}$ such that

$$
\int_{0}^{\alpha_{2} \circ \gamma(\varepsilon)} \frac{d s}{\kappa_{1}(s)} \leq \frac{T_{\varepsilon}}{1+\kappa_{2}\left(\varrho\left(\varepsilon, T_{\varepsilon}\right)\right)}<+\infty
$$

where $\kappa_{1}, \kappa_{2} \in \mathcal{K}$ as in (16). Moreover,

$$
T\left(x_{0}\right) \leq T_{\varepsilon}
$$

for $\left\{x_{0} \in \mathbb{R}^{n}:\left|x_{0}\right| \leq \varepsilon\right\}$.

Remark 3 If the presented conditions are satisfied for the set $\left\{x_{0} \in \mathbb{R}^{n}: V\left(x_{0}\right) \leq c\right\}, c \in \mathbb{R}_{>0}$ then the system (1) is locally OFTS.

Example 1 Consider the system

$$
\begin{gathered}
\dot{x}_{1}=-\frac{\left\lfloor x_{1}\right\rceil^{0.5}}{1+g\left(x_{2}\right)}, \\
\dot{x}_{2}=x_{2}
\end{gathered}
$$

with $y=x_{1}$.

I. Let $g\left(x_{2}\right)=\ln \left(1+\ln \left(1+\left|x_{2}\right|\right)\right)$. Choose the function $V$ as $V=\left|x_{1}\right|^{1.5}$. The function $V$ satisfies (9) and (6) with $\lambda(x)=\ln \left(1+\ln \left(1+\left|x_{2}\right|\right)\right)$ since

$$
\begin{aligned}
& \dot{V}(x)=-1.5 \frac{V^{2 / 3}(x)}{1+\ln \left(1+\ln \left(1+\left|x_{2}\right|\right)\right)}=-1.5 \frac{V^{2 / 3}(x)}{1+\lambda(x)}= \\
& -\alpha_{3}(V(x), \lambda(x))
\end{aligned}
$$

and (9) is satisfied for $\alpha_{3}(s, r)=1.5 \frac{s^{2 / 3}}{1+r}$. Since $\left|x_{2}(t)\right|=$ $\left|x_{2}(0)\right| e^{t}$ we have $\lambda(x) \leq \ln \left(1+\ln \left(1+\left|x_{2}(0)\right| e^{T}\right)\right)=$ $\varrho\left(\left|x_{2}(0)\right|, T\right)$ for $t \in[0, T]$. Then, considering (19) we obtain the inequality

$$
2 \sqrt{\varepsilon}\left(1+\ln \left(1+\ln \left(1+\left|x_{2}(0)\right| e^{T_{\varepsilon}}\right)\right)\right) \leq T_{\varepsilon},
$$

that always has a solution for any $x_{0} \in \mathbb{R}^{n}$. Thus, the system is globally OFTS.

II. Let $g\left(x_{2}\right)=\left|x_{2}\right|$. Then for $V=\left|x_{1}\right|^{1.5}$ we have

$$
\dot{V}(x)=-1.5 \frac{V^{2 / 3}(x)}{1+\left|x_{2}\right|}=-1.5 \frac{V^{2 / 3}(x)}{1+\lambda(x)}=-\alpha_{3}(V(x), \lambda(x))
$$

with $\lambda(x)=\left|x_{2}\right|$. Since $\left|x_{2}(t)\right|=\left|x_{2}(0)\right| e^{t}$ we have $\lambda(x) \leq\left|x_{2}(0)\right| e^{T}=\varrho\left(\left|x_{2}(0)\right|, T\right)$ for $t \in[0, T]$. Then, considering (19) we obtain the inequality

$$
2 \sqrt{\varepsilon}\left(1+\left|x_{2}(0)\right| e^{T_{\varepsilon}}\right) \leq T_{\varepsilon} .
$$

It is obvious that this inequality has a solution for sufficiently small $\left|x_{0}\right|$, i.e. the system is locally OFTS. For example, the solution is $T_{\varepsilon} \in[2.18,5.01]$ with $x_{0}=(1,0.01)^{T}$, and $T\left(x_{0}\right) \leq 2.18$ (see Fig. 1).

Remark 4 Note that the presented results can be used for some systems with OS-Lyapunov function. For example,

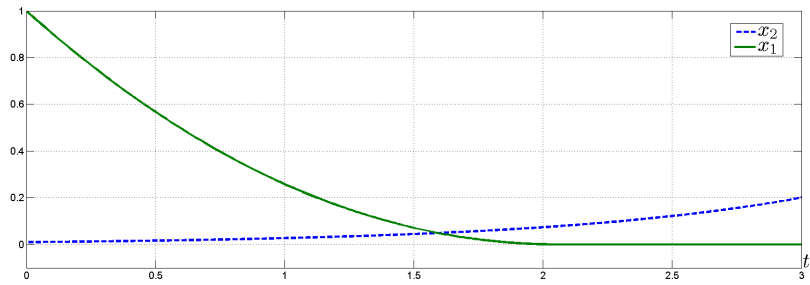

Fig. 1. System states versus time

considering the system (21), (22) with $y=\sin x_{1}$ the function $V=\left|x_{1}\right|^{1.5}$ is not OLOS-Lyapunov function since (9) is not satisfied. However, due to the output $y=x_{1}$ is FTS (see Example 1) it is obvious that the system with $y=\sin x_{1}$ is also OFTS.

It is worth to highlight that in the presented results the settling-time function might be discontinuous. In [18] it is shown that for the case of state finite-time convergence the settling-time function $T(x)$ is continuous if and only if it is continuous at 0 . It is not satisfied for output stability in general case.

Example 2 Consider the system

$$
\begin{aligned}
& \dot{x}_{1}=-\frac{\left\lfloor x_{1}\right\rceil^{0.5}}{1+\left|x_{2}\right|}, \\
& \dot{x}_{2}=-x_{2},
\end{aligned}
$$

I. Let $y=x_{1}$. The system is OFTS and $T\left(x_{0}\right)=$ $\ln \left(\left(\left|x_{2}(0)\right|+1\right) e^{2 \sqrt{\left|x_{1}(0)\right|}}-\left|x_{2}(0)\right|\right)$ is continuous.

II. Let $y=x_{1} x_{2}$. Since $x_{2}(t)=0$ only if $x_{2}(0)=0$, then the system is OFTS with $T\left(x_{0}\right)=$ $\ln \left(\left(\left|x_{2}(0)\right|+1\right) e^{2 \sqrt{\left|x_{1}(0)\right|}}-\left|x_{2}(0)\right|\right)$ for $x_{2}(0) \neq 0$ and $T\left(x_{0}\right)=0$ for $x_{2}(0)=0$, i.e. the settling-time function is discontinuous at $\left(x_{1}, 0\right), x_{1} \in \mathbb{R} \backslash\{0\}$.

In the paper [17] it is shown that in the case of partial stability for the system (11) the settling-time function $T\left(x_{10}, x_{20}\right)$ is (jointly) continuous if and only if it is continuous at $\left(0, x_{2}\right)$.

The next results are presented for SIOS class of systems.

Corollary 3 Consider the SIOS system (1). Let a SIOSLyapunov function satisfies the inequality

$$
\dot{V}(x) \leq-c V(x)^{\mu}
$$

for all $x \in \mathcal{X}$. Then the system (1) is OFTS. Moreover, the settling-time function satisfies

$$
T(x) \leq \frac{1}{c(1-\mu)} V(x)^{1-\mu} .
$$

Example 3 Consider the system

$$
\begin{gathered}
\dot{x}_{1}=-\left(2+\sin \left(x_{2}\right)\right)\left\lfloor x_{1}\right\rceil^{0.5}, \\
\dot{x}_{2}=x_{2}
\end{gathered}
$$

with $y=x_{1}$. Let $V=\left|x_{1}\right|^{1.5}$. The function $V$ is SIOSLyapunov function due to $\dot{V}(x)=-1.5\left|x_{1}\right|\left(2+\sin \left(x_{2}\right)\right) \leq$ $-1.5 V^{2 / 3}$. Then the system is globally OFTS and $T\left(x_{0}\right) \leq$ $2 V\left(x_{0}\right)^{1 / 3}$. 


\section{CONCLUSIONS}

In the paper necessary and sufficient Lyapunov characterizations of output finite-time stability are presented for the class of OLOS and SIOS systems (1). The presented stability analysis of OFTS opens a lot of topics for future research. For example, an extension to the class of OS systems, control and observer design.

\section{REFERENCES}

[1] H.G. Kankanamalage, Y. Lin, Y. Wang, On notions of input-tooutput stability for nonlinear systems with time delays, Proc. of IEEE International Conference on Control and Automation, pp. 490-495, 2017.

[2] G. Wang, X. Zhang, S. Mei, S. Wu, Input-to-output stability analysis of interconnecting microgrids, Proc. of 4th International Conference on Electric Utility Deregulation and Restructuring and Power Technologies, pp. 1097-1103, 2011.

[3] H.G. Kankanamalage, Y. Wang, Lyapunov descriptions of robust output stability for systems with delays, Proc. of 55th Conference on Decision and Control, pp. 6416-6421, 2016.

[4] R.G. Sanfelice, Results on input-to-output and input-output-to-state stability for hybrid systems and their interconnections, Proc. of the IEEE Conference on Decision and Control, pp. 2396-2401, 2010.

[5] D. Efimov, A. Loria, E. Panteley, Robust output stabilization: Improving performance via supervisory control, International Journal of Robust and Nonlinear Control, vol.21(10), pp. 1219-1236, 2011.

[6] J. Liu, M. French, Robust stability of input-output systems with initial conditions, SIAM Journal on Control and Optimization, vol. 53(3), pp. 1625-1653, 2015.

[7] V.I. Vorotnikov, Yu.G. Martyshenko, On partial stability theory of nonlinear dynamic systems, Journal of Computer and Systems Sciences International, vol. 49(5), pp. 702-709, 2010.
[8] C. Jammazi, Continuous and discontinuous homogeneous feedbacks finite-time partially stabilizing controllable multichained systems, SIAM Journal on Control and Optimization, vol. 52(1), pp. 520-544, 2014.

[9] V. Vorotnikov, Partial stability, stabilization and control: some recent results, in 15th IFAC Triennial World Congress, 2002.

[10] C. Jammazi, On a sufficient condition for finite-time partial stability and stabilization: applications, IMA Journal of Mathematical Control and Information, vol. 27(1), pp. 29-56, 2010.

[11] D. Angeli, E.D. Sontag, Forward completeness, unboundedness observability, and their Lyapynov characterizations, Systems \& Control Letters, vol. 38, pp. 209-217, 1999.

[12] S.N. Dashkovskiy, D.V. Efimov, E.D. Sontag, Input to State Stability and Allied System Properties, Automation and Remote Control, vol. 72, no. 8, pp. 1579-1614, 2011.

[13] B. Ingalls, Y. Wang, On Input-to-Output Stability for Systems not Uniformly Bounded, Proc. NOLCOS, St. Petersburg, July 2001.

[14] E.D. Sontag, Y. Wang, Lyapunov Characterizations of Input to Output Stability, SIAM J. Control Optim., no. 39(1), pp. 226-249, 2000.

[15] E.D. Sontag, Y. Wang, A Notion of Input to Output Stability, Proc. Eur. Control Conf., Brussels, July 1997, DOI: 10.23919/ECC.1997.7082720.

[16] E.D. Sontag, Y. Wang, Notions of Input to Output Stability, Syst. Control Lett., no. 38(4-5), pp. 235-248, 1999.

[17] W.M. Haddad, A. L'Afflitto, Finite-time partial stability and stabilization, and optimal feedback control, Journal of the Franklin Institute, vol. 352, pp. 2329-2357, 2015.

[18] S.P. Bhat, D.S. Bernstein, Finite-time stability of continuous autonomous systems, SIAM J. Control Optim., vol. 38, no. 3, pp. $751-$ 766, 2000.

[19] F. Amato, R. Ambrosino, C. Cosentino, G. De Tommasi, Input-output finite time stabilization of linear systems, Automatica, vol. 46(9), pp. $1558-1562,2010$ 